\title{
Comparison of Electric, Thermal and Combined Treatment Effect on Solid- Liquid Extraction
}

\author{
Samia Ben-Ali ${ }^{\# 1}$ \\ ${ }^{\#}$ Research laboratory of engineering processes and industrials systems, \\ National Engineering School of Gabes, University of Gabes, St Omar Ibn El-Khattab, 6029 Gabes, Tunisia \\ ${ }^{1}$ samia.benali@enig.rnu.tn
}

\begin{abstract}
The purpose of this work is to compare the effect of the pulsed electric field (PEF), the thermal and the combined treatment on liquid-solid extraction process by studying mass transport within biological materials (sugar beets). Adouble envelope treatment chamber of solid liquid extraction on a laboratory scale is conceived allowing simultaneous measurements of the soluble matter concentration and electric conductivity of the material during the extraction process. Different temperatures are studied in the range of $\left[25-60^{\circ} \mathrm{C}\right]$. The effectiveness of temperature increase is studied in terms of the Brix and mass of sugar variation before and after the treatment. Pulsed electric field (PEF) is applied as pretreatment and intermediate treatment during the extraction process with a strength of 150, 250 and $750 \mathrm{~V} / \mathrm{cm}$. The influence of PEF on conductivity and diffusion coefficient before and after treatment $\left(\mathbf{D}_{a v}\right.$ and $D_{a p}$ ) is currently being investigated. A comparison between the efficiency of the thermal, the electrical and the combined treatment on solid-liquid extraction shows that the effectiveness of the soluble matters diffusion increases with electric field intensity and temperature increase. Nevertheless, combined treatment is the best in term of energy consumption.
\end{abstract}

Extraction, Diffusion, Pulsed electric field, Thermal treatment, Combined treatment.

\section{INTRODUCTION}

The preservation of food, both fresh and processed, has always been one of the main problems of human history $[1,2,3,4,5,6]$. There are several traditional methods of processing. The most used and most known is the thermal treatment $[1,7,8]$. This treatment is encountered in most parts of food industry such as drying, sterilization and extraction. The solid-liquid extraction is the fundamental operation that aims to extract, separate or dissolve, by immersion in a liquid or by spraying with a liquid, one or many components (solid or liquid). Extraction processes for fruit and vegetable juices constitute an important part of food industry. The process used to extract sugar beet juice is composed of many stages (slicing, extraction, purification, evaporation and crystallization) where the extraction is a key step influencing all the transformation process. Conventionally, sugar beet slices are first rapidly preheated, to denature cells membranes, for 10 min at high temperature $\left[80-90^{\circ} \mathrm{C}\right]$. Juice extraction is then carried by counter-current diffusion using distilled water at 70 ${ }^{\circ} \mathrm{C}$ for 80 to $90 \mathrm{~min}$. Nevertheless, this treatment induces cells damage and adversely affects nutritional compounds such as vitamins, pigments, polyphenols, or antioxidant compounds. Furthermore, degradation reactions are accelerated by temperature increase [9]. This procedure is very costly in terms of energy and generates impurities in the juice which complicates enormously the stages of the process especially purification and crystallization steps. Moreover, it affects the juice quality regarding its nutritional value and its aspect $[9,10$, 11]. In order to improve the juice quality, many researches are focused so far on the alternative extraction process $[12,3,13,14,6]$. One of these alternative treatments is the application of pulsed electric field treatment. It acts by transforming and breaking the fruit cells, thus increasing the conductivity and permeability of the materials, this effect is known as electroplasmolysis. This phenomenon can be explained by two factors: (1) electroporation that means the enlargement of the pores (2) the denaturation of the membrane cells resulting from the ohmic heating caused by the membranes electrical resistance. [15] stated that cell destruction is due solely to temperature. He has made some measurements indicating that the elevation of the conductivity during an electrical treatment is due to the increase of the temperature of the sample. However, subsequent work has shown that electrical treatment can ensure permeabilization without heating [16]. PEF treatment has been used to enhance alfalfa juice extraction efficient [17], drying of foods combined to osmotic dehydration of apple, carrot and banana [18], extraction of bioactive compounds of pumpkin [6], extraction of red beetroot pigment $[12]$ and others applications $[19,16]$. The aim of this work is to compare the PEF, the thermal and the combined treatment on efficiency of sugar beet juice extraction. 


\section{MATERials AND Methods}

An easy way to comply with the conference paper formatting requirements is to use this document as a template and simply type your text into it.

\section{A. Preparation of Sample}

Sugar beets were washed, cut into rectangular slices with the dimensions $4 \mathrm{~cm} \times 5 \mathrm{~cm} .10 \mathrm{~g}$ of fresh beets are taken and placed in an oven at $105^{\circ} \mathrm{C}$ in a container, which is already dried and weighed. The moisture content of beet slices is given by the formula:

$$
M=\frac{m 2-m 3}{m 2-m 1} * 100
$$

$m_{1}$ : mass of the empty container, $m_{2}$ : mass of container + mass of fresh sample, and $m_{3}$ : mass of container + mass of dry sample. $20 \mathrm{~g}$ of fresh sugar beets are placed in the extraction chamber between two electrodes connected to the pulsed field generator. The distilled water circulated with a flow rate of $3 \mathrm{~g} / \mathrm{min}$ on the sample. Extracted juice was recuperated at the outlet of the extraction chamber every two minutes.

\section{B. Experimental Apparatus}

The experimental apparatus shown by figure $\underline{1}$, consisted of a double envelope polypropylene cell with two electrodes connected to a PEF generator ( manufactured by Electrical Engineering Department at International Engineering School of Gabes in Tunisia). The distance between electrodes is variable. Distilled water circulates continuously through the extraction chamber using a peristaltic pump. Concentration measurements were based on Brix ( $\mathrm{g}$ of solute/100 $\mathrm{g}$ of solution). Values were obtained using a digital hand refractometer (ATAGO PR101, ATAGO Co., Japan). This set-up is used to perform a combined electrical and thermal treatment. Heated water circulate in a double envelope of the treatment chamber containing sugar beet slices to be treated at the desired temperature. The temperature inside the cell is measured using a thermocouple; this temperature must be the same as that of the solution. The Brix varies with the temperature, thus juice must be cooled to room temperature before making Brix measurements.

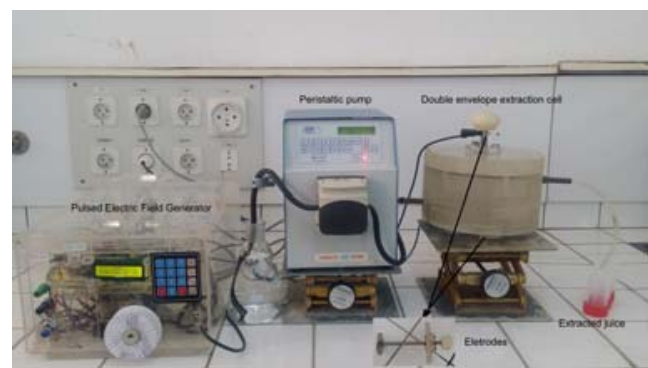

Fig. 1. : Experimental apparatus for PEF and thermal treatment

\section{Brix Measurement}

Brix measurement ( $\mathrm{g}$ of solute/ $100 \mathrm{~g}$ of solution) was performed in two ways: every two minutes at the outlet of the extraction chamber after treatment and every five minutes of accumulate extracted solution. The sugar fractions thus obtained are used to follow the sugar evolution concentration in the liquid and solid phase versus time and then estimate the diffusion coefficient. The initial Brix of sugar beet slices varies from 19.9 and 22.1 (g of solute/g of solution).

\section{Electrical Measurement}

The experimental electrical treatment parameters are the potential intensity, the pulse number, the pulse duration and the repetition period. During the experiments of intermediate electrical treatment or pre-treatment, we have fixed: The repetition period: $10 \mathrm{~ms}$, pulse duration: $100 \mathrm{~ms}$, pulse number: 1000 . For intermediate treatment, the pulsed electric field is applied after $50 \mathrm{~min}$ of extraction when the mass transfer becomes constant. The electrical intensities used during the experiments are 150,250, and $750 \mathrm{~V} / \mathrm{cm}$. The conductivity, describing the ions transfer, were measured using an OHAUS conductimeter model ST3100C. To ensure the conductivity measurements, the two electrodes were connected to a computer controlled pulse generator; the control software is used to measure the resistance before and after the electrical treatment as well as the voltage and current.

\section{E. Measurement of Color}

In order to study the quality of extracted juice, we proceeded to determine the absorbance of the sugar solutions recovered every five minutes. We evaluate the effect of the electrical and/or thermal treatment on the juice color by mea- suring the transmittance of light beam for the wavelength $640 \mu \mathrm{m}$. The light transmittance is measured from 0 to $100 \%$ compared to a reference (pure water). The clarity of the obtained juice after the electrical and thermal treatment was defined according to the absorbance of light energy. 


\section{III.RESULTS AND DISCUSSIONS}

\section{Text Font of Sugar beet MEB characterization}

\section{A. Sugar Beet MEB Characterization}

Scanning Electron Microscopy identifies the cellular components of sugar beets. Figure 2 represents the structure of a plant cell whose main constituents are the cell wall and protoplasm [20]. This includes the cytoplasm, vacuole and nucleus. Cells and membranes are defined by an important parameter for the determination of the electrical properties. It is the characteristic diameter that varies between $10 \mu \mathrm{m}$ and $200 \mu \mathrm{m}$ for biomaterials, for sugar beet it is of $200 \mathrm{~m}$ whose cytoplasm seems to be uniform.

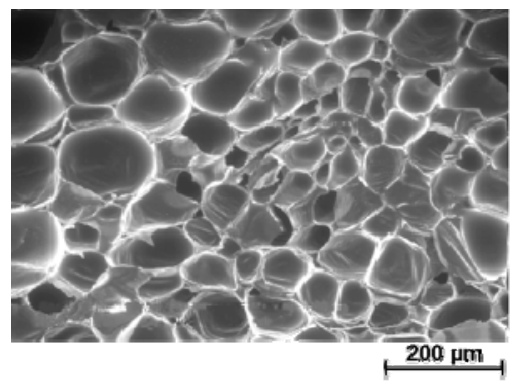

Fig. 2. Scanning Electron Microscopy of sugar beet membranes [21]

\section{B. Diffusion coefficient}

Diffusion is occurred in two ways: Diffusion in the beet slices itself, i.e. the displacement of the soluble substances from the interior of slices to its surface and the diffusion in the liquid phase, i.e. the passage of solute from the beet slices surface into the liquid phase. The determination of the diffusion coefficient is based on the first and second Fick laws represented by the following equations $(2,3)$ :

$$
\begin{gathered}
\partial C / \partial t=D_{a} \partial^{2} C / \partial x^{2} \\
-D \partial C / \partial x=k\left(C-C_{0}\right)=\frac{1}{A} \frac{\partial N}{\partial t}
\end{gathered}
$$

The solution of these equations is given by Newman (1931) for three geometrical forms: Cube, cylinder and sphere (equations 4, 5 and 6). The average dimensional concentration is obtained by integration respect to position and time respectively. Newman showed that results given for the cubic form are used to obtain the solution for a parallelogram and for a cylinder of infinite length [22].

For cube:

$$
\frac{\bar{C}-C_{0}}{C_{1_{0}}-C_{0}}=\frac{8}{\pi^{2}} \sum_{n=0}^{\infty} \frac{1}{(2 n+1)^{2}} \exp \left[-(2 n+1)^{2}\left(\frac{D t}{a^{2}}\right)\left(\frac{\pi}{2}\right)^{2}\right]
$$

For sphere:

$$
\frac{\bar{C}-C_{0}}{C_{1_{0}}-C_{0}}=\frac{6}{\pi^{2}} \sum_{n=1}^{\infty} \frac{1}{n^{2}} \exp \left[-n^{2}\left(\frac{D t}{a^{2}}\right) \pi^{2}\right]
$$

For cylinder:

$$
\frac{\bar{C}-C_{0}}{C_{1_{0}}-C_{0}}=4 \sum_{n=1}^{\infty} \frac{1}{R_{n}^{2}} \exp \left[-\left(\frac{D t}{a^{2}}\right) R_{n}^{2}\right]
$$

where $\mathrm{R}_{n}$ is the root of $\mathrm{J}_{0}(\mathrm{x})$

$$
J_{0}(x)=1+\sum_{n=1}^{\infty}(-1)^{n} \frac{x^{2 n}}{(2 n)^{2} !}=0
$$

The equation 2 is used to calculate diffusion coefficient. Considering that the transfer is unidirectional and the sugar concentration in the slices is assumed to be evenly distributed and initially equal to $\mathrm{C}_{0}$. To resolve equation 2, initial and limit conditions are required and defined as:

Initial conditions:

$$
C=C 0 \quad ;-L<x<+L, \quad \grave{a} \quad t=0
$$


The boundary conditions such as at the boundary between the beet slices and the solution $(x= \pm L)$ the sugar rates are the same in the inlet of beet slices and the outlet of solution, thus:

$$
\alpha \partial C / \partial t= \pm D \partial C / \partial x ; \quad x= \pm L, \quad \grave{a} t>0
$$

The solution of this equation is:

$$
\frac{M_{t}}{M_{\infty}}=1-\sum_{n=1}^{\infty} \frac{2 \alpha(1+\alpha)}{1+\alpha+\alpha^{2} q_{n}^{2}} \exp \left(-D q_{n}^{2} t / l^{2}\right)
$$

where the $\mathrm{q}_{n}$ are the positive roots of:

$$
\tan q_{n}=-\alpha q_{n}
$$

It should be noted that $\alpha=a / K l$, it is convenient to express the fractions of the concentrations in terms of $(\alpha)$. At equilibrium the total amount of solute in the solution and in the slices are initially contained in the initial solution concentration $\mathrm{C}_{0}$.

$$
\frac{\alpha C_{\infty}}{K}+l C_{\infty}=a C_{0}
$$

or $C_{\infty}$ is the final concentration in the solid or in the juice according to the transfer of sugar from the solid to the juice or vice versa. The final quantity of sugar, $M_{\infty}$ is given by:

$$
M_{\infty}=2 l C_{\infty}=\frac{2 a C_{0}}{1+a / l K}=\frac{2 a C_{0}}{1+\alpha}
$$

The final fraction of the sugar obtained in the juice is given by:

$$
\frac{M_{\infty}}{2 a C_{0}}=\frac{1}{1+\alpha}
$$

For example if $50 \%$ of the initial solute in the solution is finally in the slices, $\alpha=1$. In the particular case of a quantity of infinite solute $\alpha=\infty$, the roots of the equation $\underline{11}$ are:

$$
\frac{M_{t}}{M_{\infty}}=1-\frac{8}{\pi^{2}} \sum_{n=0}^{\infty} \frac{1}{(2 n+1)^{2}} \exp \left[-D\left(n+\frac{1}{2}\right)^{2} \pi^{2} t / l^{2}\right]
$$

in terms of concentration this equation 15 is written:

$$
\frac{\bar{C}-C_{\infty}}{C_{0}-C_{\infty}}=\frac{8}{\pi^{2}} \sum_{n=0}^{\infty} \frac{1}{(2 n+1)^{2}} \exp \left[-D\left(n+\frac{1}{2}\right)^{2} \pi^{2} t / l^{2}\right]
$$

In the case where all the surfaces of the slices participate in the diffusion, the global solution of the Fick law is simply the product of the three solutions corresponding to each direction:

$$
\tau=\frac{8}{\pi^{2}} \exp \left(-\frac{1}{L^{2}} D\left(\frac{1}{4}\right) \pi^{2} t\right)
$$

$\tau$ is the ratio of juice concentration at time $t,\left(\mathrm{C}_{t}\right)$ and initial concentration $\left(\mathrm{C}_{0}\right), l$ is the equivalent sugar beet slices dimensions. $\tau$ is computed experimentally to determine the diffusion coefficient $D$.

\section{Influence of Electrical Treatment on Concentrations}

To illustrate the effect of the PEF, we have studied different voltage intensities $(150,250$ and $750 \mathrm{~V} / \mathrm{cm})$. We observe that the diffusion process accelerates and the juice concentration increases when applying PEF at $50 \mathrm{~min}$. Figure 3 represents the variation of Brix ( $\mathrm{g}$ of solute/100 $\mathrm{g}$ of solution) accumulation as a function of time for the different fields studied. We note that all these experiments are repeated at least twice and the results represented in the figures are the averages of the different repetitions. According to this figure, we notice the clear variation of the concentration after application of the electric field this variation depends on its intensity. We note that extraction yield is improved when we increase electric field intensity. It is clear that the increase of the intensity of the electric field generates a clear efficiency of the extraction. Based on the above measurements, the variation of the concentration into the beet slices bed can be deduced and is represented in Figure 4 . The cumulative mass of sugar over time is calculated from mass measurements of juice recovered after five minutes at a constant rate of $3 \mathrm{~g} / \mathrm{min}$, which is the product of Brix by the mass of the solution and is represented on the figure 5 . 


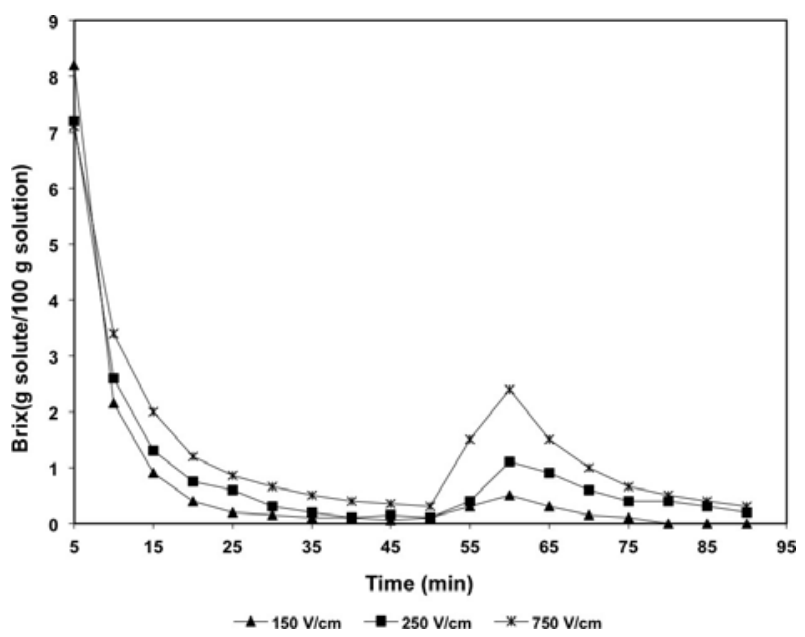

Fig. 3. Brix variation of extracted juice

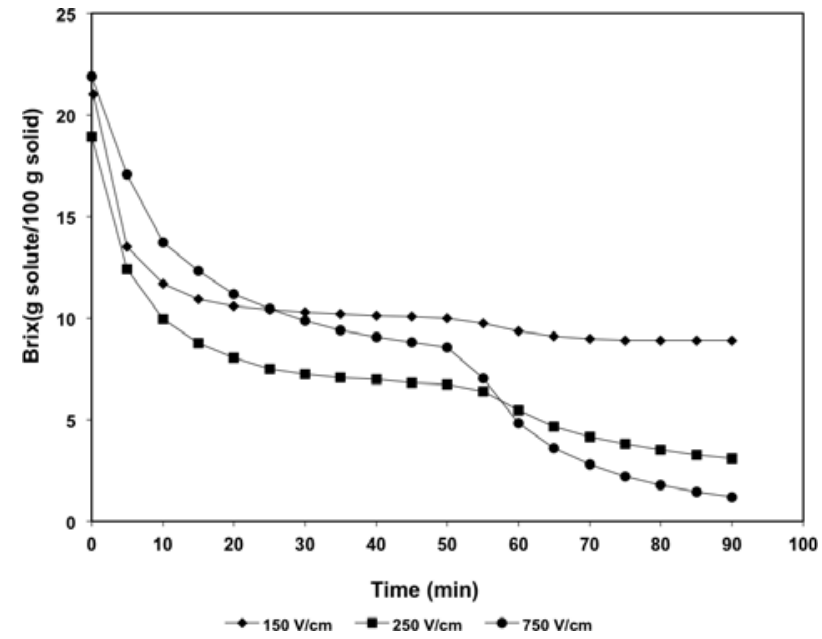

Fig. 4. Brix variation into the beet slices bed

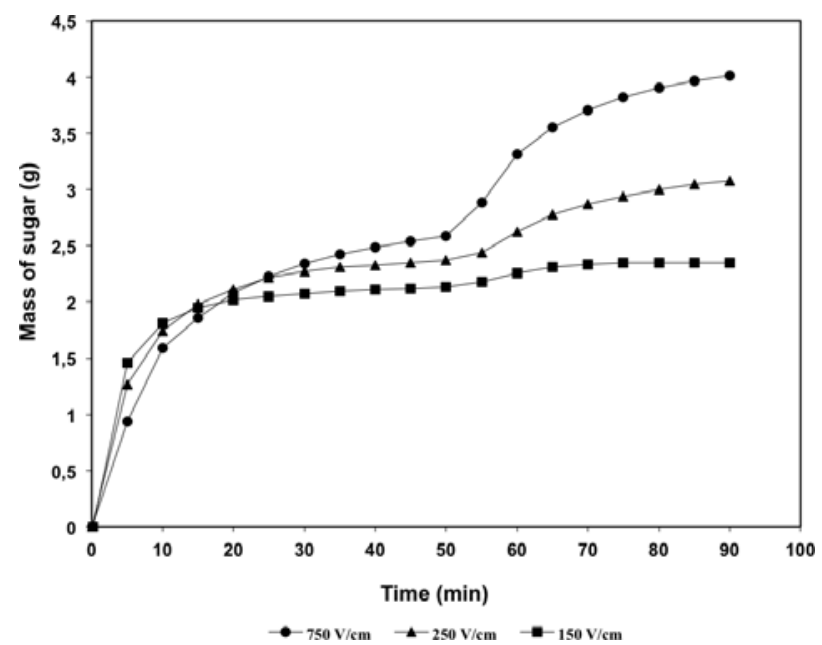

Fig. 5. Cumulative mass of sugar in solution 
The efficiency of the electrical treatment is expressed in terms of the conductivity before and after treatment. The ions transfer is more important for high PEF intensity. From figure $\underline{6}$, electrical treatment is clearly noted from the sharp increase in conductivity which marks the destruction of cell barrier against the transfer of matter. From this result, conductivity measurement may be used to study matter diffusion variation during extraction.

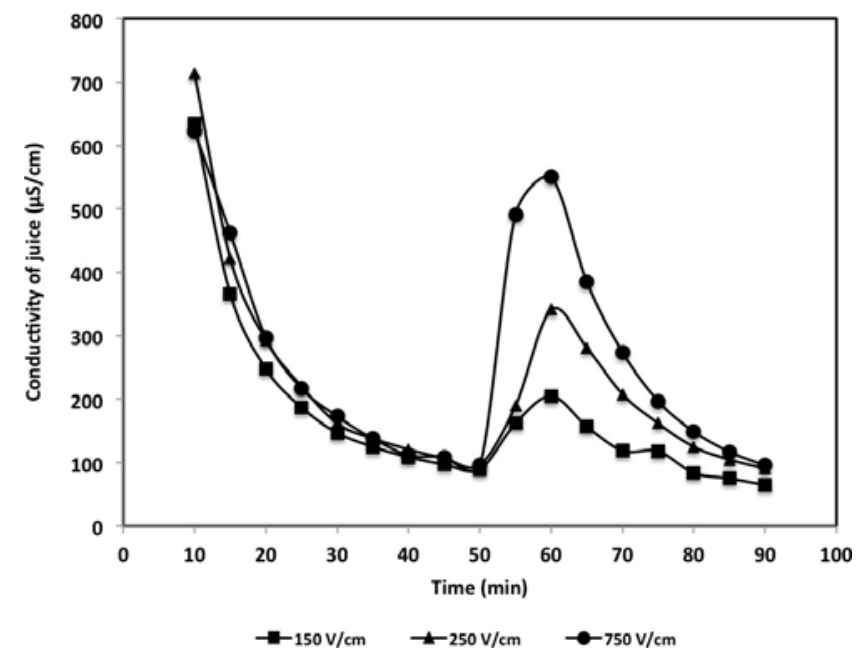

Fig. 6. Effect of PEF treatment on the conductivity of sugar beet juice

The efficiency of the PEF for three studied voltage intensities $(150,250$ and $750 \mathrm{~V} / \mathrm{cm})$ is expressed in term of diffusion coefficient, which is calculated as described in section $B$. The obtained results are presented in table 1 .

TABLE I. Diffusion coefficient before and after PEF application

\begin{tabular}{|l|l|l|}
\hline PEF (V/cm) & $\mathbf{D}_{a v}\left(\mathbf{1 0}^{-\mathbf{1 0}} \mathbf{m}^{2} \mathbf{s}^{-\mathbf{1}}\right)$ & $\mathbf{D}_{a p}\left(\mathbf{1 0}^{-\mathbf{9}} \mathbf{~ m}^{\mathbf{2}} \mathbf{- \mathbf { 1 }}\right)$ \\
150 & & 1.35078 \\
250 & $7.50431 \pm 0.167$ & 11.4066 \\
750 & & 28.5164 \\
\hline
\end{tabular}

\section{Effect of Pulsed Electric Field Pretreatment}

Figure 7 represents the variation of the concentration within the bed of beet slices versus time basing on Brix measurements,. This variation depends on the pulsed electric field intensity. The more the latter is increased, the more the extraction is improved. By application of the pre-electrical treatment, the diffusion process accelerates and the concentration of the juice increases. The increase of the intensity of the electric field gives a clear efficiency of the extraction. The cumulative mass of sugar over time is calculated from mass measurements of juice recovered after five minutes at a constant flow rate of $3 \mathrm{~g} / \mathrm{min}$, which is the product of Brix by the mass of the solution and is represented in the figure 8.

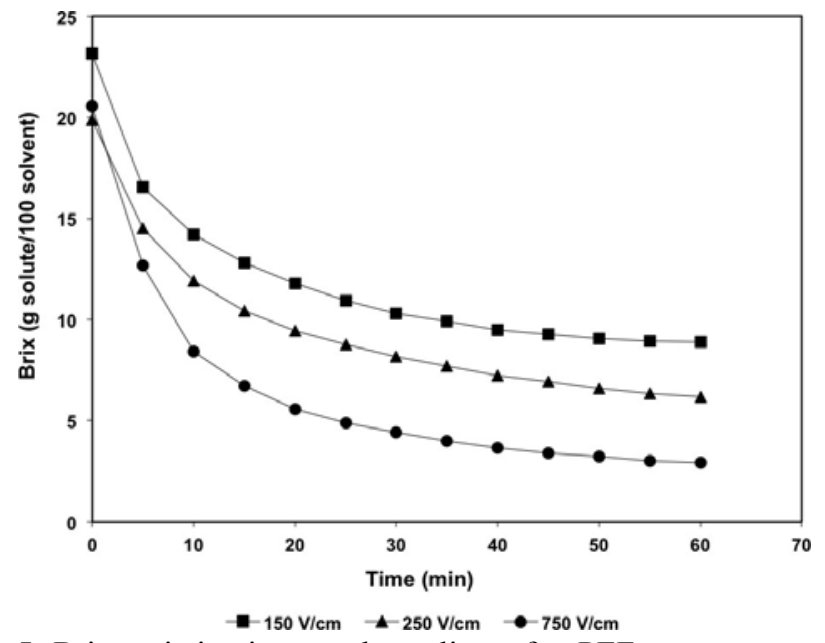

Fig. 7. Brix variation in sugar beet slices after PEF pre-treatment 


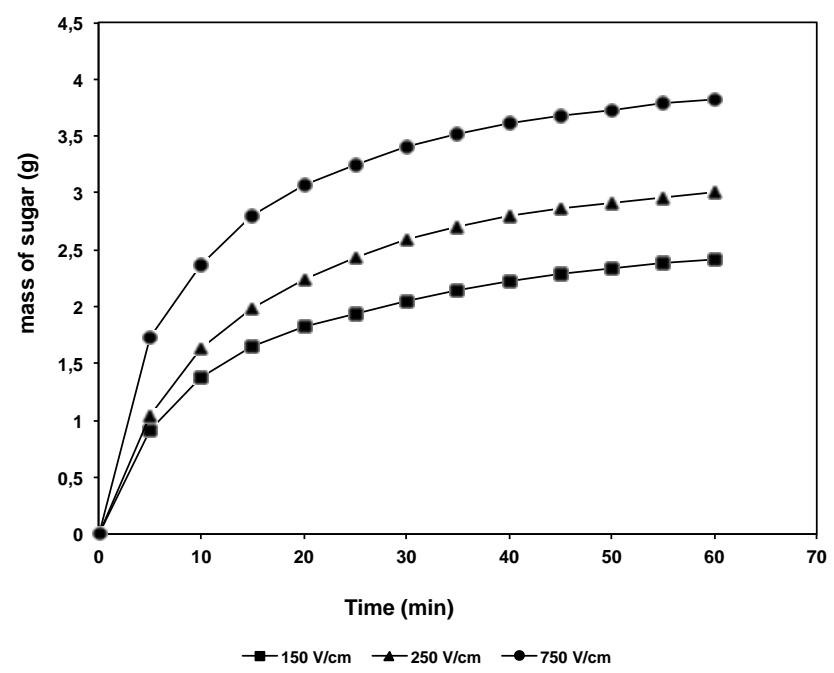

Fig. 8. Cumulative mass of sugar during extraction after PEF pre-treatment

\section{E. Effect of Thermal and combined Treatment}

The purpose of this study is to combine both types of electrical and thermal treatment to minimize the negative effects of very high temperature heat treatment and power consumption by decreasing the density of the field. We then carried out experiments by combining an intermediate electrical treatment for a constant density of $750 \mathrm{~V} / \mathrm{cm}$ and at variable temperatures $\left(25^{\circ} \mathrm{C}, 35^{\circ} \mathrm{C}, 50{ }^{\circ} \mathrm{C}\right.$ and $\left.60{ }^{\circ} \mathrm{C}\right)$. During these experiments, we heated the beet slices at the desired temperature. The distilled water used for extraction is also heated. The temperature is controlled inside the extraction chamber and the water using a thermocouple. The effect of the electrical treatment is appreciable at low temperatures. When temperature increases, the effectiveness of the treatment becomes negligible as shown in Figure 9. The cumulative sugar mass over time is calculated from the juice mass measurements recovered after five minutes for studied temperatures (figure 9). It is noted that the intermediate electrical treatment is very detectable for the temperatures $25^{\circ} \mathrm{C}$ and $35^{\circ} \mathrm{C}$. Whereas, for $60{ }^{\circ} \mathrm{C}$, sugar concentration decreases. This can be explained by beet slices denaturation due to high temperature.

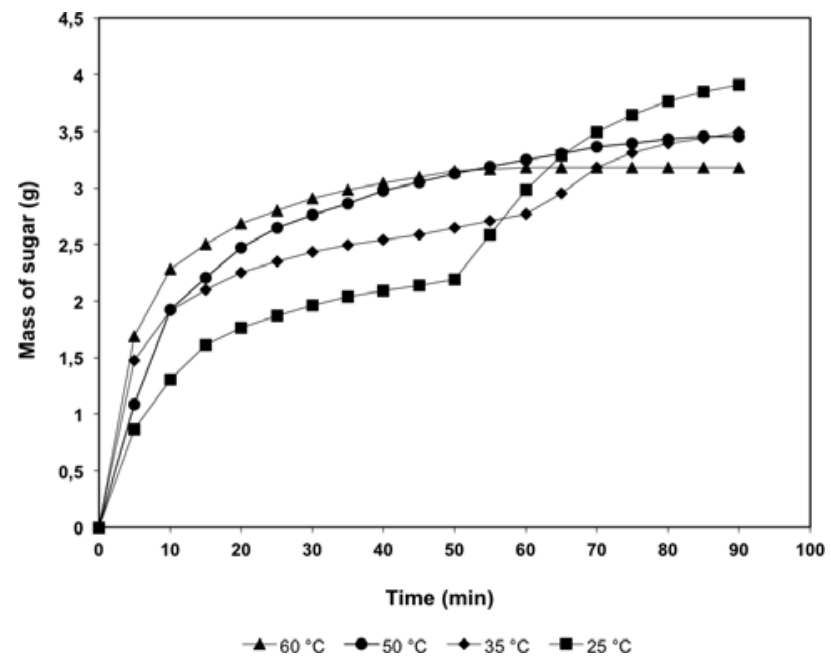

Fig. 9. Effect of temperature on cumulative mass of sugar extracted

The application of an intermediate electrical treatment or a pre-treatment has a beneficial effect on the diffusion of matter. An improvement both quantitative and qualitative is obtained after an electrical treatment. In addition, a review of energy consumption reveals that a $100 \%$ electric treatment has relatively high consumption, to reduce this consumption we used a combined electrical and thermal treatment at moderate temperature (figure 10). 


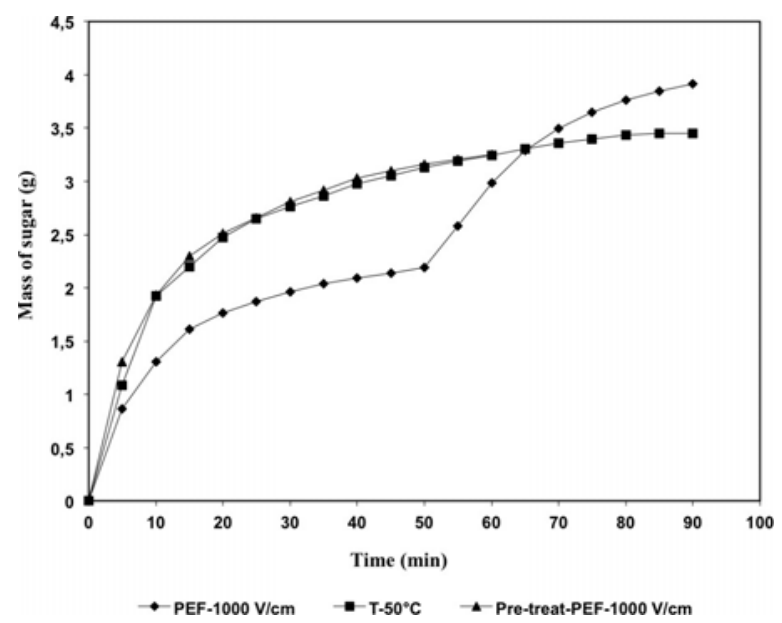

Fig. 10. Effect of temperature on cumulative mass of sugar extracted

\section{F. Qualitative Characterization}

In order to study the quality of the obtained juice by thermal and electrical treatment, we proceeded to determine the transmittance of the sugar solutions recovered every five minutes at wavelength of $640 \mu \mathrm{m}$ Figure 11 shows the effect of both applied treatments on the juice color. Photometric measurements showed that a clearer juice is generally obtained after the PEF treatment. We think that under the effect of such a treatment; a considerable part of the components of the cellular membrane (e.g. pectin) as well as the substances responsible for the coloring of the juice are retained in the structure of sugar beet. These substances are much more present in the juices resulting from a thermal extraction.

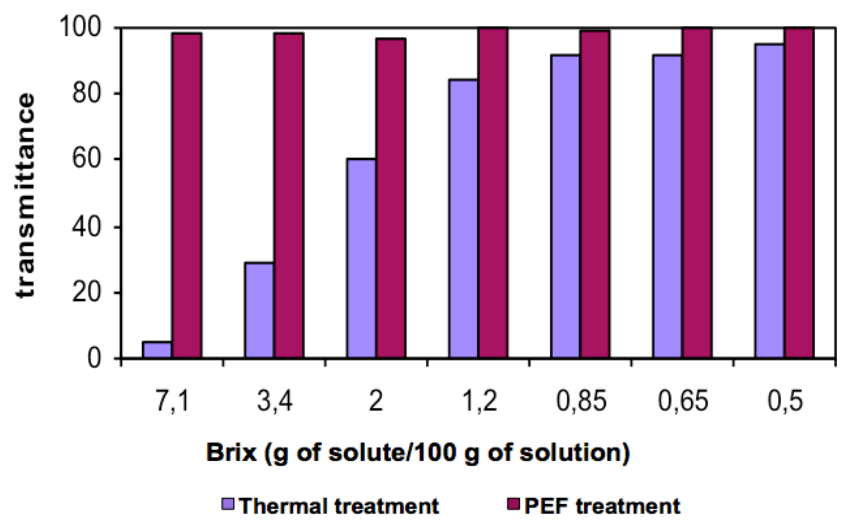

Fig. 11. Transmittance of the juice obtained by thermal and PEF treatment

\section{IV.CONCLUSION}

The aim of this work is to study of the effect of thermal or/and electrical treatment by PEF on solid-liquid extraction process. The obtained results show that the diffusion after a PEF treatment, thermal or combined is improved. The effectiveness of treatment has been studied in terms of Brix in juice of beet slices, also in term of the cumulative mass of sugar and the diffusion coefficients before and after treatment $\left(\mathrm{D}_{a v} / \mathrm{D}_{a p}\right)$. The diffusion of soluble materials increases with the intensity of the electric field. This is because such treatment intensifies the permeability of the cellular membranes without the damage as compared to a heat treatment at high temperature $\left(70^{\circ} \mathrm{C}\right)$. Nevertheless the PEF at moderate temperature may be more efficient, especially in term of energy consumption. Photometric measurements show that the extracted juice obtained after an electrical treatment is clearer than that obtained from thermal one

\section{ACKNOWLEDGMENT}

This work was supported by the Ministry of the Higher Education and Scientific Research in Tunisia.The

\section{REFERENCES}

[1] L. Ludikhuyze, a. V. Loey, M. Hendrickx, K. Universiteit, Combined high pressure thermal treatment of foods, Woodhead Publishing Lim- ited, 1914. URL: http://dx.doi.org/10.1533/9781855736610.3.266. doi:10.1533/9781855736610.3.266.

[2] R. Moreira, A. M. Sereno, Evaluation of mass transfer coefficients and volumetric shrinkage during osmotic dehydration of apple using sucrose solutions in static and non-static conditions, J. Food Eng. 57, 25-31, 2003.

[3] L. N. Cook, Comparisons of physicochemical properties of watermelon juice, 1-54, 2009.

[4] B. Bchir, Contribution à l'étude de conservation Des Graines De Grenade ( Punica Granatum L .) Par D'eshydratation Osmotique, Ph.D. thesis, academie universitaire wallonie-europe univer- site de lie'ge gembloux agro-bio tech, 2011. 
[5] H. Ando, K. Kajiwara, S. Oshita, T. Suzuki, The effect of osmotic de- hydrofreezing on the role of the cell membrane in carrot texture softening after freeze-thawing, J. Food Eng. 108, 473-479, 2012.

[6] J. Garc'ia-Parra, F. Gonz'alez-Cebrino, J. Delgado-Ad'amez, R. Cava, O. Mart'in-Belloso, P. Elez-Mart'inez, R. Ram'irez, Application of innova- tive technologies, moderate-intensity pulsed electric fields and high-pressure thermal treatment, to preserve and/or improve the bioactive compounds content of pumpkin, Innov. Food Sci. Emerg. Technol. 45, 53-61, 2018.

[7] S. Stefancich, F. Delben, Interaction of food proteins with polysaccharides, II. Properties upon freeze-drying of mixed solutions and thermal treatment of the lyophilizate, J. Food Eng. 31, 347-363, 1997.

[8] A. A. Barba, Thermal treatments of foods: A predictive general-purpose code for heat and mass transfer, Heat Mass Transf. und Stoffuebertragung 41, 625-631, 2005.

[9] M. Asadi, Beet-Sugar Handbook 59, 2006.

[10] H. Mhemdi, O. Bals, E. Vorobiev, Combined pressing-diffusion technology for sugar beets pretreated by pulsed electric field, J. Food Eng. 168, 166-172, 2017.

[11] F. Almohammed, H. Mhemdi, E. Vorobiev, Purification of juices obtained with innovative pulsed electric field and alkaline pressing of sugar beet tissue, Sep. Purif. Technol. 173, 156-164, 2017.

[12] Y. Chalermchat, M. Fincan, P. Dejmek, Pulsed electric field treatment for solid liquid extraction of red beetroot pigment : mathematical modelling of mass transfer 64, 229-236, 2004.

[13] A. Rawson, A. Patras, B. K. Tiwari, F. Noci, T. Koutchma, N. Brunton, Effect of thermal and non thermal processing technologies on the bioactive content of exotic fruits and their products: Review of recent advances, Food Res. Int. 44, 1875-1887, 2011.

[14] E. Vorobiev, N. Lebovka, Pulse Electric Field-Assisted Extraction, January. 2011. URL: http://www.crcnetbase.com/doi/abs/10.1201/ b11241-3. doi:10.1201/b11241-3, .

[15] Bruniche-Olsen, Solid liquid extraction, in: Copenhagen NYT Nord. Forl., 1962, pp. 423-433.

[16] W. Sitzmann, E. Vorobiev, N. Lebovka, Applications of electricity and specifically pulsed electric fields in food processing: Historical backgrounds, Innov. Food Sci. Emerg. Technol. 37, 302-311, 2016.

[17] T. K. Gachovska, A. A. Adedeji, M. O. Ngadi, Influence of pulsed electric field energy on the damage degree in alfalfa tissue, J. Food Eng. 95, 558-563, 2009.

[18] E. Amami, L. Khezami, A. B. Jemai, E. Vorobiev, Osmotic dehydration of some agro-food tissue pre-treated by pulsed electric field: Impact of impeller's Reynolds number on mass transfer and color, J. King Saud Univ. - Eng. Sci. 26 (2014) 93-102.

[19] F. DeVito, Application of Pulsed Electric Field (PEF) Techniques in Food Processing, Chem. Eng. 116. 2006.

[20] [K. S. Suslick, Sonochemistry, 1998. doi: 10.1002/0471238961.

[21] M. Bazhal, E. Vorobiev, Electrical treatment of apple cossettes for inten- sifying juice pressing, J. Sci. Food Agric. 80, 1668-1674,

[22] A. E. ordan, Carol A and Neumann, Eberhard and Sowers, Electroporation and electrofusion in cell biology, Springer Science $\backslash \&$ Business Media, 2013.

\section{AUTHOR PROFILE}

Samia Ben-Ali was born in Tunisia in 1975. She received her Chemical engineering diploma in 2000 from ENIG, University of Gabes, Tunisia, her Chemical engineering Master degree in 2001 from the UTC, Compiegne, France and her Ph.D. degree in 2004 from the University of Bordeaux, France. She is a member of Research laboratory of engineering processes and industrials systems, National Engineering School of Gabes, University of Gabes. She is working in the field of agro-industrial system and process and especially in the valorization of agriculture wastes and wastewater treatment. 